\title{
DIGITALTECHNOLOGY Introduction to the cognitive hospital
}

\author{
Authors: Jordi Rovira-Simón, ${ }^{\mathrm{A}}$ Marc Sales-i-Coll, ${ }^{\mathrm{B}}$ Patricia Pozo-Rosich, ${ }^{\mathrm{C}}$ Darren Gates, ${ }^{\mathrm{D}}$ Carol Patt, ${ }^{\mathrm{E}}$ \\ Iain Hennessey, ${ }^{F}$ Lisa Emery, ${ }^{G}$ Juan Antonio Hueto-Madrid, ${ }^{H}$ Marta Carbonell-Cobo, ${ }^{I}$ Francesc Garcia-Cuyàs, ${ }^{J}$ \\ Monica Moz, ${ }^{\mathrm{K}}$ Zafar Chaudry' ${ }^{\mathrm{L}}$ and Genevieve Shaw ${ }^{\mathrm{M}}$
}

\begin{abstract}
The third industrial revolution has radically impacted the transformation of hospitals. Through the adoption of key digital technologies, hospitals have become more accessible, flexible, organised, responsive and able to deliver more personalised care. The digitalisation of patient health records, one of the most remarkable achievements to date in healthcare management, has enabled new opportunities, including the idea of hospitals evolving to become artificially intelligent. In parallel, the adoption of electronic and mobile internet technologies in hospitals has introduced new structural concepts, seeing a variety of terms blossom such as 'smart', 'intelligent', 'green' and 'liquid'. Now in the early fourth industrial revolution, driven by AI and internet-of-things technologies, this article unveils a new concept adapted to the upcoming era.
\end{abstract}

KEYWORDS: cognitive hospital, smart hospital, green hospital, intelligent hospital, artificial intelligence

DOI: $10.7861 /$ fhj.2021-0016

\section{Introduction}

Digitalisation is driving an increase of hospital capacity by overcoming the inherent constraints of such large and complex

Authors: Adigital health medicine PhD student, Hospital

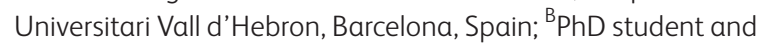
healthcare process transformation expert, Universitat de Girona, Girona, Spain; ' neurology specialist, Hospital Universitari Vall d'Hebron, Barcelona, Spain; ${ }^{D}$ paediatric intensivist and clinical innovation consultant, Alder Hey Children's Hospital, Liverpool, UK;

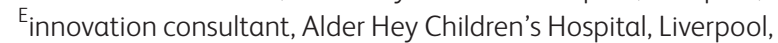
UK; ${ }^{F}$ director of innovation and paediatric surgeon, Alder Hey Children's Hospital, Liverpool, UK; ${ }^{G}$ chief information officer, The Royal Marsden NHS Foundation Trust, London, UK; ${ }^{H}$ chief of unit of oral and maxillofacial surgery and professor of surgery, Hospital Universitari Vall d'Hebron, Barcelona, Spain; ${ }^{\text {I }}$ chief information officer, Institut Català d'Oncologia, Barcelona, Spain; ' deputy medical director, Hospital Sant Joan de Déu de Barcelona (HSJD),

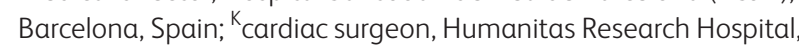
Milan, Italy; 'senior vice president and chief information officer, Seattle Children's Hospital, Seattle, USA; M $_{\text {independent editor and }}$ copywriter, Saxlingham Nethergate, UK organisations. These include poor communication and coordination across big, fragmented premises and numerous teams; limited access to updated, timely clinical and operational information in data systems; and, especially, fragmented siloed data. $^{1}$

In recent decades, the increasing adoption of digital technology, including a wide take-up of key enabling electronic medical record (EMR) solutions, advanced medical devices and equipment to improve diagnostics and treatments, as well as other key information systems, has radically expanded the limits of healthcare and our understanding about the role of hospitals. ${ }^{2}$

The recent explosion of the internet-of-things (IoT) technologies has only accelerated the exponential growth of data in clinical facilities as a result of the rapid uptake of a multitude of sensors and devices and the integration of their data streams, together with those provided by the expanding myriad of systems and solutions in the hospital. ${ }^{3}$ In turn, this has paved the way for the start of AI solutions in this sensitive domain, which promise to enable a proactive healthcare based on predictive and, later, prescriptive algorithms. ${ }^{4}$ While the first step is for predictive analytics to provide forecast capabilities, the next step is for prescriptive algorithms to generate automated recommendations. ${ }^{5}$ Likewise, this transformation will drive greater personalisation of care and precision medicine by the addition of genomics medicine data and technology, while also embracing the increasing social call to achieve greener and accessible hospitals. ${ }^{6}$

Every new technological development in recent decades has helped spur the terms 'smart hospital' and 'intelligent hospital' to portray a more futuristic image of these facilities. Nevertheless, in practice, these terms have, so far, been focused on presenting specific applications that address local problems and challenges, rather than viewing the hospital as a global entity. ${ }^{7,8}$ The rocketing growth of data and the increasing capacity to integrate and quickly process large volumes of information through big data and AI techniques takes us to the beginning of a new era that needs new paradigms to help express what is to come, which has been named 'Society 5.0'.'

This paper aims to introduce the cognitive hospital concept, first studying the evolution of key former terms (ie 'smart hospital', 'intelligent hospital', 'green hospital' and 'liquid hospital') that introduced a futuristic view of the hospital, and subsequently reflecting on the need for this new concept. This article discusses the idea of hospitals as complex artificially intelligent entities that, as such, touch upon a whole range of ideas linked to studies of the brain and nervous system. These 
developments have the ultimate goal of encouraging the standardisation of hospitals' level of cognition in preparation for the fourth industrial revolution.

\section{Methods}

We present a survey of literature that investigates the evolution of key terms that referred to the hospital as an advanced entity. We reviewed literature published between 1990 and 2020 from the public databases of the Google Scholar search engine. Before 1990, these concepts were non-existent. The goal was to compare the number of manuscripts that used the terms 'smart hospital' and 'intelligent hospital'. To do that, we defined the search to include the right phrase, in English, at any part of the article. Citations and patents were excluded from the search. Furthermore, the increasing prevalent terms 'green hospital' and 'liquid hospital' have also been investigated as they add relevant dimensions to the future cognitive hospital definition (such as awareness, sustainability, plasticity and the physical architecture of the hospital). The difficulty in investigating these two terms was that, unlike the smart or intelligent hospital, the latter coincided with actual names of clinical interventions or even hospitals (eg Hither Green Hospital). For this reason, the search focused on looking at the first 100 most relevant works on the matter, in periods of 5 years, from 1990 to 2020.

\section{Literature survey}

\section{Smart vs intelligent hospital}

'Smart hospital' and 'intelligent hospital' are terms that have been used in recent decades to refer to the increasing cleverness shown by hospitals through the adoption of new technologies.

\section{Up to 1990}

Over the last few decades, as life has undergone a continuous process of digitalisation, first with the arrival of electronics and personal computers, then with the appearance of smartphones and now with the emergence of the IoT, all areas of society have been constantly transforming. The healthcare system has traditionally been a slow adopter of digital innovations as its critical role in public health tends towards conservative behaviour. However, this has not stopped new concepts from transforming hospitals over time. As informatics started to be widely adopted by the general public in the late 1980s, the first publications using the term 'smart hospital' began to appear. ${ }^{10}$

\section{$1991-2000$}

Interestingly, the expression 'intelligent hospital' appeared a few years later in the early 1990 s as, predominantly, researchers in Japan started to understand the power of medical informatics for transforming the healthcare system by piloting the very first EMR solutions. ${ }^{11,12}$ From then on, the term 'intelligent hospital' appeared to gain traction as more publications used it (ie 44 references compared with 21 mentioning 'smart hospital' from 1991-2000). This leading position was driven by the link with soft technological areas (such as 'information systems', 'messaging solutions', 'knowledge-based solutions', 'middleware' and 'agent software components') versus the association with a more hardware-based perception and the term 'smart', which in its early days was strongly linked to location technologies and sensors. ${ }^{13}$

\section{1-2010}

Over the following decade (2001-2010), the same dynamic can be observed in the first 5 years (ie 66 new references compared with 42 using 'smart hospital'), both terms maintaining their initial links with software and more technological-hardware inventions. Without a doubt, this was the decade of the internet and the onset of the world wide web (WWW). Therefore, concepts related to data accessibility (such as 'unified modelling language (UML)', 'ontology', 'semantics' and 'ubiquitous computing') started to be more popular and were also connected to the 'intelligent hospital' concept. On the contrary, 'smart' was still linked to advanced hardware and physical innovations involving 'sensor networks', 'embedded systems' and 'smart spaces'. Interestingly, a publication mentioning both terms ('The intelligent hospital: patient smart card') reinforces our perception that 'smart' was reserved for a more technical innovation while 'intelligent' was used as a broader concept, referring to a hospital that embraces technical innovations. ${ }^{14}$

Over the last 5 years of that decade, however, the strong adoption of radio-frequency identification (RFID) and mobile technologies started to boost the appearance of the 'smart' concept with references to 'advanced location', 'tracking' and 'activity monitoring' solutions, surpassing the intelligent hospital by 294 vs 173 citations. $^{15}$ The use of 'intelligence' in the period 2006-2010 intensifies the mixture of emerging terms (including 'business process', 'semantic management', 'information exchange' and 'context awareness') as the Google revolution truly transforms access to vast amounts of data and these concepts start to be applied in the digitalisation of patients' clinical records. ${ }^{16}$

\section{$2011-2020$}

The start of the new decade confirms the explosion of the 'smart' concept and the standstill of the 'intelligent hospital' as broadband and mobile technologies conquer all areas of life and are mostly associated with the term 'smart'. In the years 2011-2015, 'smart' surpasses 'intelligent' by 561 references to $247 .{ }^{17}$ This confirmed lead is driven by the emergence of remote monitoring pilots, their sensors and wearables. However, in the same period, 'intelligent' begins to be more associated with the first applications flourishing in healthcare (with names such as 'intelligent operating room', 'intelligent appointment', 'automated pharmacy' and 'mHealth') and appears to be a term used to denote a more ethereal concept that goes beyond technology. ${ }^{18}$ Over the following 5 years, 'smart' carries on growing exponentially in use through the disruption driven by the IoT concept, surpassing the use of 'intelligent hospital' by 2,040 references to 277 , through the decisive launch of the use of sensors. ${ }^{19}$ This last push appears to reformulate the links with the word 'smart' that has also come to encompass software concepts (such as new AI, cloud, blockchain and augmented reality (AR) technologies) and even final solutions (such as scheduling, security and telehealth services). Nevertheless, the use of 'intelligent' decelerates as 'smart' takes over its original associations, although it still retains a special link with big data and data-driven concepts. Today, more general media uses 'smart' as the most common term that embraces concepts referring to an advanced futuristic hospital. 
The smart hospital sidelines the intelligent hospital by embracing key concepts from IoT and AI.

\section{Green hospital}

'Green hospital' is the term used to refer to a hospital that is eco-friendly. This concept has been driven by the adoption of new technologies in recent decades.

Up to 1990

This term was completely unknown in literature.

$1991-2000$

With less intensity than the previous concepts, this term introduces the physical dimension of the hospital, its architecture, use of clean energies, reduction of water use and management of waste (such as anaesthetic gasses, which have a significant impact on the greenhouse effect). The utilisation of the concept 'green hospital' appears following the Kyoto Protocol in 1997, and the first references can be found from then on. ${ }^{20,21}$

\section{$2001-2010$}

The term starts to take off between 2001-2005, with four publications focused on green architecture with a strong lead in the USA, which saw the first hospital calculating its ecological footprint. ${ }^{22}$ From 2006-2010, after the Montreal Action Plan focused on the extension of the Kyoto Protocol, continued interest increased in the topic, with 27 references adding new subjects (such as energy control systems, and heating, ventilating and air conditioning solutions (HVAC)) driven by the significant contribution of Asian countries and the construction of the first green hospital in the USA. ${ }^{23}$

\section{$2011-2020$}

The term seems to reach peak growth during the period 20112015, with 59 references which now include works all around the globe (ie USA, China, Japan, Taiwan, European countries, India, Iran etc). At this time, it reaches global acceptance and gains more emphasis in relation to engineering aspects, such as the installation of light-emitting diode (LED) lighting and more efficient HVAC solutions, which lends a considerable technological aspect to the concept. From 2016-2020, the term reaches 67 references, driven by the Paris Climate Agreement, yet its growth appears to slow. These references include more sophisticated connotations, added to by IoT technologies, such as intelligent energy and HVAC systems. ${ }^{24}$ Interestingly, the renewed hopes of the new decade seems to be an acceleration of the number of works using the term 'green hospital' due to greater social awareness of the topic highlighted by the campaigning of Greta Thunberg and the increasing pressure on public authorities to stop climate change. This is symbolised by new policies such as the European Green Deal or UK's 25-year plan to improve the environment. ${ }^{25,26}$ Likewise, the appearance of $\mathrm{AI}$ in this topic becomes a reality. ${ }^{27}$

The green hospital is at the brink of exponential growth due to an increase of climate change awareness and AI technologies.

\section{Liquid hospital}

'Liquid hospital' is a term that refers to the accessibility of care that goes beyond the hospital's walls to reach a patient's home. It was coined by Sant Joan de Déu hospital in Barcelona and has become prevalent in general media, with thousands of articles in search engines. However, it has still not thrived in the scientific domain with barely five publications from 2015-2020. ${ }^{28}$ Nevertheless, we felt that it is was important to include it in this article as it adds another dimension related to plasticity that will enrich the future definition of the cognitive hospital.

The term clearly leverages the ideas of 'liquid modernity' proposed by Zygmunt Bauman, where the philosopher establishes that current society does not have rigid boundaries anymore and that public and private domains are in a constant blend. ${ }^{29} \mathrm{As}$ a result of the increased improvement of communications and availability sensors and applications, this term moved to portray hospitals as an extended seamless and invisible network of applications able to overcome the hospital's physical boundaries, enabling some care at home.

The liquid hospital will extend care outside the walls of a traditional hospital, engaging patients anywhere.

\section{Results}

From the previous analysis, it can be quickly spotted that the 'smart hospital' concept has taken over, with over 2,000 publications from 2015-2020, compared with 277 publications of 'intelligent hospital' (Fig 1). IoT and AI are the main drivers of this growth.

The concept of the 'green hospital' grew quickly after the Montreal Action Plan came into effect, supported by more sophisticated energy control systems and eco-friendly HVACs technologies. Interestingly, from 2015-2020, the term appears to fall in growth (Fig 2). Recent climate change awareness seems to have boosted the concept again.

\section{Discussion}

There is a need of a new overall term to face the future of the artificially intelligent, green and boundaryless hospital.

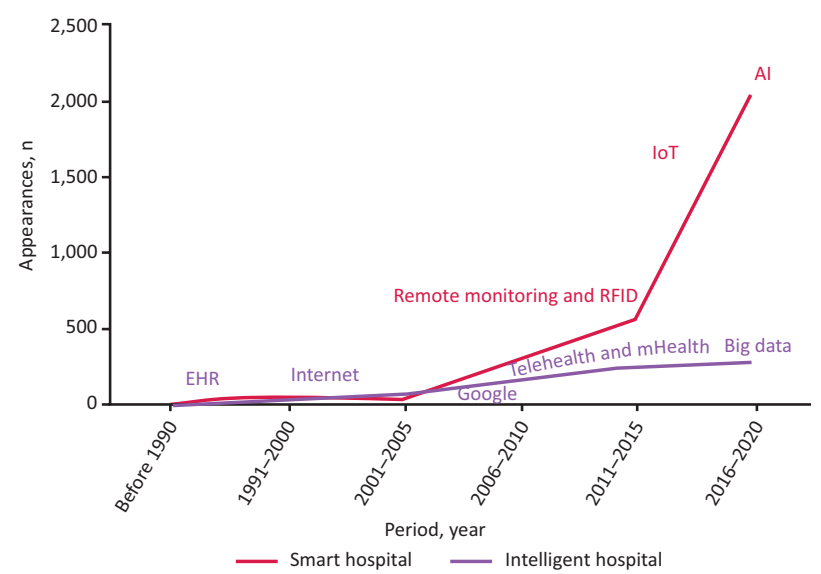

Fig 1. Publications containing 'smart hospital' and 'intelligent hospital' $\mathrm{AI}=$ artificial intelligence; $\mathrm{EHR}=$ electronic health record; $\mathrm{IOT}$ = internet of things; $\mathrm{RFID}=$ radio-frequency identification. 


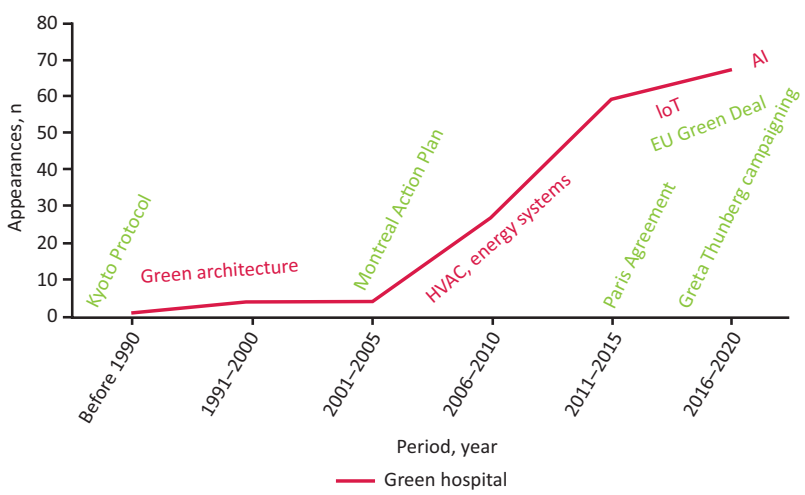

Fig 2. Publications containing 'green hospital'. $A I=$ artificial intelligence; HVAC = heating, ventilating and air conditioning solutions; IoT $=$ internet of things.

The last 2 decades have been heavily dominated by the adoption of EMR solutions, which is one of the key enablers in the hospital digitalisation journey that spurs the use of the terms 'smart' and 'intelligent'. AI policy in medicine and its application has advanced at pace over the past 5 years alongside a global commitment to a greener planet. This means that a hospital of the future now needs to be cognisant of autonomous capabilities that provide healthcare in a responsible and sustainable way, to benefit all of society.

Cognitive studies could help develop a robust new model for the upcoming AI era by leveraging a neuroscience background. ${ }^{30}$

\section{What is cognition when applied to a hospital?}

Cognition is the ability to process information from perception and senses, acquired knowledge (experience) and the subjective characteristics that allow the information to be assessed. ${ }^{31}$ It consists of processes such as learning, reasoning, attention, memory, problem solving, decision making and feelings, all of which are optimised by the nervous system to obtain results with the minimum expense of energy. All of this is smart, green and probably liquid, among other adjectives; a cognitive hospital, though, is also one that learns, adapts and connects with professionals.

We could imagine the hospital of the future as an entity, able to learn from the environment, integrating staff talent, and interacting with patients and professionals to personalise care on a daily basis. How then can a hospital, a complex rigid physical structure with hundreds of healthcare professionals, transform into an intelligent adaptive entity in the broader sense of the word?

Similar to the nervous system, the cognitive hospital would be distributed across functional levels and physical areas to enable a wide range of applications that are still connected to a common 'being', the hospital 'brain'. This intelligence could follow an organisation similar to the human body, going from the smallest component, the cell, to the tissue, then the organ, the system and finally the organism. This might resemble the hospital organisation going from the professional to the team, then the service, the department and, finally, the hospital as whole. Every single level is vital and autonomous. It develops local intelligence and is coordinated with its peers and upper structures with the leading role of the brain, as shown in Fig 3. This hierarchisation is also found in several approaches that interpret the human brain and nervous system as a whole. These approaches can be used to shape the cognitive hospital concept, which might also help devise its architectural design on a physical and functional level from varying perspectives:

> placing emphasis on physical cognitive distribution: central and peripheral

> focusing on level of awareness to execute actions: somatic and autonomous

> dividing the nervous system between receptors and actuators: sensitive and motor.

These ways of representing the nervous system can be extrapolated to depict the cognitive hospital concept. This could be roughly defined as an artificial body that will seamlessly orchestrate healthcare provision by resembling the cognitive capacities of the human brain and the rest of the nervous system. As such, this involves the integration of a multitude of local activities (eg processes and pathways in the emergency department (ED), intensive treatment unit (ITU), wards, theatre suites, hospitalisation, outpatient clinics, administration, logistics and procurement) and intelligences (eg individual expertise and decisions by staff, and AI provided by medical devices and monitoring systems) to generate overall meanings and insights to control global processes in the hospital, in real time. Likewise, this global awareness will support healthcare staff in their specific, local, operational tasks by providing the big picture at all times to enable the best decision making possible to optimise healthcare outcomes, including patient and staff satisfaction.

This way of performing mimics the hierarchy of the human body. Staff represent the essentials cells of the hospital, working in a coordinated fashion as teams. These teams, symbolised as tissues in Fig 3, are highly specialised and implement very specific tasks according to their role in the service (ie ED, surgery, ITU etc). The best performance of any service or organ requires perfect orchestration and communication between each different layer, which is conducted by the 'hospital brain'. A good delivery of care requires a fine coordination of varying services, and, in the same way that our body handles the digestive, respiratory and circulatory systems, the cognitive hospital should manage urgent and elective care so that the experience in each department is optimal for patients, families and staff. Patients and families will benefit from a seamless delivery of care, avoiding unexpected disruptions (ie waiting times or cancellations) and receiving constant and adequate information. Staff will be helped by
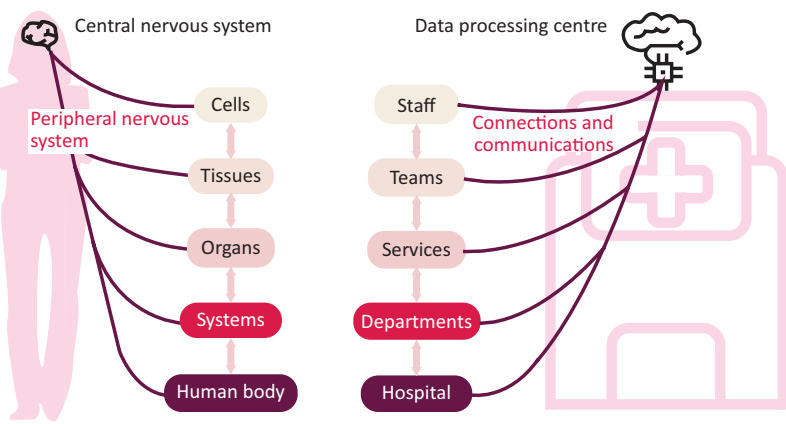

Fig 3. Comparing the human nervous system with the cognitive hospital. 
real-time applications and devices that provide meaningful insights to support optimal clinical conditions, decision making and communication between teams, services and departments.

The following subsections propose a brief description about how the anatomic and digital world can be blended by the presented concept.

\section{The hospital's central and peripheral nervous system} On a higher level, the nervous system's architecture is divided into the central nervous system (CNS), composed of the brain and spinal cord, and the peripheral nervous system (PNS), containing nerves that reach all parts of the body and connections with the CNS.

Interestingly, this anatomical model fits well with the physical distribution of intelligence in a hospital building. In fact, the anatomical model could be extrapolated to the infrastructure, the physical elements that make up a body. As happens with the human body, the central nervous system is mainly composed of the brain and the spinal cord. Similarly, a hospital's 'brain' is located in the data centre, while the spinal cord is the main channel that enables communication with the different peripheral areas that comprise a hospital. Likewise, the peripheral nervous system that is composed of a myriad of nerves, ramified to reach every part of the body, could be modelled as the various communication channels, cables and different networks that reach every single area in a hospital, where digitalisation enables data generation (sensors), information gathering and processing and, eventually, data-driven actions to close the intelligence loop.

For instance, in a critical, time-sensitive, clinical pathway (such as the stroke code), all the healthcare professionals involved (from the paramedics in the ambulance and the ED team receiving the patient to the specialist intervention staff) can be represented as vital cells of the hospital body that are connected to its peripheral nervous system by means of different medical devices, sensors, diagnostic tests and communications. This constant communication should be seamlessly integrated by the hospital CNS to anticipate and streamline the coordination of services to execute a responsive intervention. The stroke pathway coordinator should be empowered by key, real-time clinical and process indicators as the patient moves along the pathway, advising and providing decision support suitably to complete the case and optimising healthcare outcomes.

Another interesting take on this concept, in line with the 'liquid hospital', is how the PNS could be highly distributed within the same hospital and even other centres (eg hub/spoke, tertiary and secondary care centres, expert communication / remote surgery etc). Furthermore, the PNS could be part of the patient's home, as an extended tentacle, that allows real-time monitoring, self-management and communication applications to carefully monitor the patient.

The hospital's somatic and autonomous system Awareness is a key factor to enable human cognition. While the autonomic system is in charge of processes without any conscious control (for actions such as cardiac function, breathing rate and many activities carried out by smooth muscle and glands), the somatic system is in charge of the actions that require awareness (such as conscious control of muscles and the skeleton during physical activity). In the same way, it could be suggested that a cognitive hospital should have these two levels of behaviour. There are many processes in a hospital that are not core for patient care provision, but which are still critically essential and foundational for higher complex functions and applications. For instance, hospital climatisation is an important task that has an impact on patient and professional satisfaction and care, in addition to hospital expenditure on electricity due to heavy HVAC solutions. The cognitive hospital should cater for this process smartly, in the background, without the need of a human operator regularly adapting the conditions for each area.

\section{The hospital's sensitive and motor system}

Another way to represent the nervous system is by means of an architecture connected to a large sensory receptor network with the motor system. The human body, like any multicellular animal, must monitor and maintain a constant internal environment, as well as monitor and respond to the external environment. In many animals, these two functions are coordinated by two integrated and coordinated organ systems: the nervous system and the endocrine system. While the nervous system coordinates rapid responses to external stimuli, the endocrine system controls slower, longer lasting responses to internal stimuli.

From a technological perspective, spurred on by the IoT and the AI explosion, hospitals will carry on implementing an increasing ubiquitous network of sensors and actuators. Thus, the cognitive hospital should integrate in real time vast amounts of data provided by its pervasive sensory system, digesting relevant datasets to generate highly contextualised responses to the range of applications provided (output), eg regulation of oxygen administered according to the level of oxygen in blood detected in patients or automatically allocating healthcare resources according to predicted healthcare demand, examples that provide staff with insightful and timely information to support the best clinical or operational decision to maximise quality of care for patients.

\section{An initial proposal for defining the cognitive hospital}

At this point, an obvious question would be to ask whether a cognitive hospital is just a facility that implements a series of AI applications. This is the scenario today with an increasing number of fragmented smart applications in the field of diagnostics, treatments and health management and care delivery. While, certainly, the achieved local smartness is already part of the very early stages of the cognitive hospital, the new concept expands exponentially in different dimensions represented by human cognition (ie learning capacity, communication/interpretation and empathy, perception, memory, anticipation/planning and problem-solving, and superior skills such as self-awareness and reflection).

This opens up the possibility of creating a model based on an entity similar to the human nervous system, inspired by its cognitive skills, hence the analogy in the earlier section. This seamless, pervasive body will integrate the distributed vast number of professional talents and capacities as well as the generated real-time data streams provided by all kinds of sensors and applications to empower staff with the right insightful information, knowledge and decision support, in the right place, at the right time, according to the specific level of patient care, process or pathway. In turn, this resembles how the human body functions, with billions of autonomous body cells cooperating, by 
means of the brain coordinating a specific organ (ie department or process in the hospital) to handle concurrent higher level activity processes or pathway.

On a different level, the new concept could enable further developments to model the increase of a hospital's intelligence according to the dimensions of human cognition. As a result, a new scaled structure could be created and subsequently standardised to enable a vision of the cognition level achieved by hospitals globally. This is similar to the process followed to monitor the level of digitalisation of hospitals in the last 2 decades with the Electronic Medical Record Adoption Model (EMRAM). ${ }^{32}$ The model developed by the Healthcare Information and Management Systems Society (HIMSS) has become a universally recognised maturation model of a hospital's EMR. As more hospitals reach the top digitalisation scale, they will be prepared for the next leap, which is where the concept presented herein can help them be prepared for the new era ahead.

\section{Conclusion}

Currently at the dawn of the fourth industrial revolution, the drive of AI and IoT technologies are leading our world to the brink of another important change: the development of cognitive skills by artificial machines and systems. Hospitals as whole bodies will be no exception, as they are increasingly adopting more advanced devices, sensors, equipment and services that are opening new horizons in areas such as diagnostics, treatments, healthcare management and delivery, hence the need to pinpoint this new concept, which embraces all the meanings of the investigated terms but also adds wider scope and powerful potential to study the level of artificial cognition of a hospital. As these technologies become more interconnected with the hierarchical organisation and processes (ie professional, team, service, department and hospital), there will be an increasing, artificially intelligent, global awareness that orchestrates all levels of care within the hospital. This process can be similar to the one followed by the brain and the nervous system when they interact with the human body. In both cases, either cells or health professionals are the key vital units, that play an autonomous role as part of a high complex organisation (ie cell, tissue, organ, system and body vs professional, team, service, department and hospital).

Digitalisation in recent decades has encouraged us to define new and clever behaviours in hospitals by using the terms 'smart' and 'intelligent'. The same digitalisation enabled the concept of the 'liquid hospital' by removing traditional physical barriers between the patient's home and the hospital to allow remote care. Likewise, digital technology has been a key enabler for the explosion of environmentally responsible technology and the appearance of the 'green hospital' concept. Overall, this digitalisation process has built the key components to enable AI functionality as part of hospital infrastructure.

Any cognitive hospital will have to implement essential cognitive capacities, and this will be supported by the consolidation of advanced artificial intelligence, IoT, 'big data' and the latest interaction and automation technologies. As soon as fast integration of vast amounts of data generated by a hospital (currently at the level of several petabytes and soon reaching the next level, exabytes) is feasible, the foundation of a common cognitive body will be possible. This will be insufficient, however, as the instant discovery of knowledge will also have to be enabled by advanced big data technologies and this, in turn, will unlock fast learning by advanced artificial intelligence algorithms. The IoT will help to nurture the perception of the being, bringing all the senses together, which will be essential to enable humanlike interaction with the hospital. However, new advanced natural language communication, voice assistants and gesture interpretation technologies will need to be in place. Right now, we are at the very early stages of these solutions, which have started to raise important ethical issues, such as who is listening to our conversations or are we being listened to all the time.

As more advanced cognition is reached, ethical concerns will grow as greater control is given to an artificially intelligent body that increasingly acts as a stakeholder. Indeed, privacy issues will become predominant as the level of pervasive monitoring has a direct impact on spaces where people's intimacy is paramount, enabling them to feel safe, and not listened to, seen or captured. Beyond these early ethical preoccupations, new concerns will arise as the hospital body gains awareness and superior cognitive skills. This would, in turn, pose important challenges, necessitating a philosophical discussion on how to deal with an AI that is able to self-motivate and set itself new goals. The likely involvement of this intelligent body in clinical tasks beyond administrative activities would pose a significant threat to the medical profession and necessitate further discussion to define the limits of this intelligence and its hierarchy. On what level of this hierarchy is the 'new hospital'? What limits will be set to prevent a 'singularity' from happening?

Whether we like it or not, the cognitive hospital will take shape over the coming years as, inevitably, technological developments enable technologically superior capacities and as a result of our responsible environmental role. Many benefits will arise from better, optimal, ubiquitous and more flexible care for citizens. This new hospital will implement advanced liquid concepts, as it will be able to reshape and build new organisational structures, as if made of toy plastic building blocks, automatically rearranging itself to cover detected needs. Over time, the cognitive hospital will be less of a physical place, as the structural limits of clinical activity imposed by the separation of the hospital from people's homes become blurred and malleable.

To make the most of these benefits and to address the presented risks, it is important to start multidisciplinary discussions on the subject with clinical professionals, technology experts, philosophers and political leaders. Defining a standardised scale for the cognitive hospital model will help to identify the service levels and quality assurance provided by a hospital facility, and it will also help regulate the limits of intelligence and people's rights at each stage. Let that be our next common challenge as a society. This way, we can turn the cognitive hospital into an opportunity for everyone, instead of succumbing to an inevitable future that is chaotically driven by individual interests.

This article is an icebreaker on the exploration of a novel concept. It lays the foundations of a complex idea while remaining flexible to incorporate refinements in works to come. Future developments will build upon the concepts defined herein to further elaborate what makes a hospital cognitive, while focusing on what limits there are and how we can measure them.

\section{Acknowledgements}

The leading role of the Royal Free London NHS Foundation Trust on robot process automation (RPA) technologies has motivated many ideas in this article. Another forerunning and inspirational initiative is EMRAD, 
which consists of the union of six NHS trusts in the East Midlands to deliver timely and expert radiology services to patients using a shared cloud system that allows them to work as a single team. Some ideas in this article were inspired by the European project 'Operating Room 4.0' (OR4.0), where one of the authors had the role of coordinator as part of MYSPHERA's team (grant agreement ID 812386; https://cordis.europa. eu/project/id/812386).

\section{References}

1 Dobrzykowski D, Tarafdar M. Understanding information exchange in healthcare operations: Evidence from hospitals and patients. Journal of Operations Management 2015;36:201-14.

2 Sood HS, McNeil K. How is health information technology changing the way we deliver NHS hospital care? FHJ 2017:4:117-20.

3 Bolhasania M, Mohsenib M, Rahmanic AM. Deep learning application for IoT in health care: A systematic review. Informatics in Medicine Unlocked 2021;23:100550.

4 Davenport T, Kalakota R. The potential for artificial intelligence in healthcare. FHJ 2019;6:94-8.

5 Lopes ], Guimarães T, Santos M F. Predictive and Prescriptive Analytics in Healthcare: A Survey. Procedia Computer Science 2020;170:1029-34.

6 Tennison I, Roschnik S, Ashby B et al. Health care's response to climate change: a carbon footprint assessment of the NHS in England. Lancet Planetary Health 2021;5:e84-92.

7 Guinard PF. Building a smart hospital using RFID technologies. In: Stormer H, Meier A, Schumacher M (Eds), European Conference on eHealth. Gesellschaft für Informatik, 2006:S131-42.

8 Sutherland J, Heuvel W. Towards an intelligent hospital environment: adaptive workflow in the OR of the future. HICSS'06 2006:100b.

9 Ioppolo G, Vazquez F, Hennerici MG et al. Medicine 4.0: new technologies as tools for a society 5.0. J Clin Med 2020;9:2198.

10 Pacione G, Vollaro G. The 'smart hospital': the role of technological structures. J Clin Computing 1988;17:17-22.

11 Sakuma S, Ishigaki T, Ikeda M. The goal of PACS in Nagoya University Hospital. Comput Methods Programs Biomed 1991;36:143-6.

12 Kondoh H, Ikezoe J, Mori Y et al. PACS in Osaka University Hospital; Computer Methods and Programs in Biomedicine. Comput Methods Programs Biomed 1994;43:57-63.

13 Beadle H, Maguire G, Smith M. Using location and environment awareness in mobile communications. Proceedings of ICICS, International Conference on Information, Communications and Signal Processing 1997:3:1781-5.

14 Fairus H. Intelligent hospital: patient smart card. Universiti Teknologi Petronas, 2004; Final Year Project.

15 Fuhrer P, Guinard D. Building a Smart Hospital using RFID technologies: Use Cases and Implementation. ECEHO6 2006.

16 Kataria P, Juric R, Paurobally S, Madani K. Implementation of Ontology for Intelligent Hospital Wards. HICSS'08 2008:253.
17 Gomez-Sacristan A, Rodriguez-Hernandez MA, Sempere V. Evaluation of quality of service in smart-hospital communications. Journal of Medical Imaging and Health Informatics 2015;5:18649.

18 Frisch P. What Is an Intelligent Hospital? A place where technology and design converge to enhance patient care. IEEE Pulse J 2014;5:10-5.

19 Uslu B Ç, Okay E, Dursun E. Analysis of factors affecting IoT-based smart hospital design. J Cloud Comp 2020;9:67.

20 Tanner R. A low energy green hospital. Health Estate J 1997;51: 2,4,6-8.

21 Beadle H, Maguire G, Smith M. Using location and environment awareness in mobile communications. Proceedings of ICICS, International Conference on Information, Communications and Signal Processing 1997;3:1781-5.

22 Germain S. The ecological footprint of Lions Gate Hospital. Hosp Q Winter 2001-2002;5:61-6.

23 Grazier K. Interview with David P Gehant, FACHE, President and Chief Executive Officer, Boulder Community Hospital. Journal of Healthcare Management 2009;54:287-90.

24 Sharma RL, Singh A. Smart energy technologies and building architecture: An overview. International Journal of Civil Engineering and Technology (IJCIET) 2019;10:473-84.

25 Breukelaar J. The European Green Deal: opportunities and challenges. JBDG EFCC, 2019.

26 Curnow J. Environmental economics in UK environmental policy: Defra's 25 Year Environment Plan. Journal of Environmental Economics and Policy 2019;8:353-8.

27 Benzidia S, Makaoui N, Bentahar O. The impact of big data analytics and artificial intelligence on green supply chain process integration and hospital environmental performance. Technological Forecasting and Social Change 2021;165:120557.

28 Aagaard A, Lindgren P. The opportunities and challenges of persuasive technology in creating sustainable innovation and business model innovation. Wireless Pers Commun 2015;81:1511-29.

29 Bauman Z. Liquid modernity. Wiley, 2013.

30 Velichkovsky BM, Ushakov VL. Cognitive science and novel medical technologies. New Concepts Reviews 2019; 8-15.

31 Baars B], Gage NM. Cognition, brain, and consciousness: Introduction to cognitive neuroscience. Elsevier, 2010.

32 Pettit L. Understanding EMRAM and how it can be used by policymakers, hospital CIOs and their IT teams. World Hospitals and Health Services 2013;49:7-9.

Address for correspondence: Jordi Rovira-Simón, Vall d'Hebron University Hospital, Passeig de la Vall d'Hebron, 119, 08035 Barcelona, Spain.

Email: jordi.roviras@e-campus.uab.cat

Twitter: @jordirosi 